\title{
Neuropsychiatric Manifestations of Neurocysticercosis
}

\author{
Nayab Mustafa Dania Al Ayyat Mazin Awad Maha Elamin Entisar Bin Haider \\ Samia Alkhoori \\ Psychiatry Department, Rashid Hospital, Dubai Health Authority, Dubai, UAE
}

\section{Keywords}

Psychiatry · Emergency · Neurocysticercosis · Neurology · Infectious disease $\cdot$ Tapeworm

\begin{abstract}
A common scenario in the Emergency Department shows patients presenting with abnormal behavior and agitation. A full workup is performed to rule out organic causes for the patients' presentation after which they are referred to the Psychiatry Department for further assessment regarding major mental disorders. Similarly, the aforementioned protocol was followed for our patient and he was admitted to the psychiatry ward but was later referred to the Neurology and Infectious Diseases Unit as he developed altered mental status. He was then diagnosed as having neurocysticercosis (NCC), which is a common parasitic infection of the central nervous system. In a multiethnic city like Dubai, NCC should always be considered as a differential diagnosis for abnormal behavior. Symptomatic NCC carries a mortality rate of more than $50 \%$, making early detection and treatment very important. Hence, it is encouraged to screen patients with a high pretest probability using brain CT and MRI.
\end{abstract}

(C) 2020 The Author(s)

Published by S. Karger AG, Basel

\section{Introduction}

Neurocysticercosis (NCC) is known for being the commonest and severest parasitic infection of the central nervous system. It is also a major public health problem in Asian, African and Latin American countries, where prevalence rates vary from 0.1 to $4 \%$. The infection is caused by the larval form of the pork tapeworm Taenia solium, which is transmitted feco-orally through eggs in contaminated water and food (especially raw vegetables, undercooked and infected pork) [1].

NCC typically first presents as seizures $(70-90 \%$ of acutely symptomatic patients) or headache. The severity of brain pathology depends on several mechanisms including quantity of cysts, type, and location as well as the host's immune response itself [2]. At the time of the first seizure, most patients have an active cyst, either a vesicle cyst or a colloid cyst. NCC is reported to be responsible for nearly half of the late-onset cases of epilepsy in the endemic areas [3].

NCC has been associated with a plethora of psychiatric manifestations such as organic psychotic conditions [4, 5], mania, schizophrenia-like symptoms or catatonic symptoms [6]. Periodic cases of NCC have been reported after assessing patients with a first episode of schizophrenia in the Western world [7]. 


\section{Case Report}

A 31-year-old Indian male, without proof of identity in his possession, was brought to the Emergency Department in Rashid Hospital by the police after he had been discovered roadside displaying abnormal behavior. He was afebrile, vitally stable and all laboratory investigations were within normal range.

He was referred to the psychiatry team for assessment. He was alert and oriented but refused to answer questions or make eye contact and exhibited psychomotor retardation. Thus, he was admitted to the psychiatry ward for further evaluation.

In the ward, the patient was not agitated or aggressive but was noted to be suspicious of others, with minimal social interaction and limited, irrelevant verbal output. He was oriented and irresponsive to the assessment of psychotic symptoms. He remained afebrile and vitally stable with no physical complaints. During his stay in the ward, he was prescribed antipsychotic medication (risperidone) for suspected brief psychotic disorder.

As the patient showed no signs of improvement by the first week, all laboratory investigations were repeated and nonenhanced CT of the brain was performed which revealed no focal lesions or hemorrhage. By the second week, he received one session of electroconvulsive therapy, after which he became clinically drowsy. Consequently, all psychotropic medications were discontinued and he was referred to the neurology team for further evaluation. Electroencephalography was performed and showed frontal intermittent rhythmic delta as well as generalized slow wave dysrhythmia with a fast sleep-like pattern. Lumbar puncture revealed a protein level of $70 \mathrm{mg} / \mathrm{dL}$, glucose $78 \mathrm{mg} / \mathrm{dL}$ and chloride $124 \mathrm{mmol} / \mathrm{L}$ for which the patient was referred to the Infectious Diseases Unit. MRI of the brain with contrast was then performed which revealed multiple ring-shaped lesions which were T1 hypointense, T2 hyperintense and hypointense on FLAIR sequences, dispersed throughout the cerebral and cerebellar parenchyma at the grey-white matter junction, with some showing mild edema (Fig. 1). Ring enhancement was noted in almost all the lesions with some showing focal intense enhancement on postcontrast study, suggestive of scolex and hence NCC in the vesicular-colloidal stage. Under care of the Infectious Diseases Unit, he was started on albendazole $400 \mathrm{mg}$ b.d. and dexamethasone 2 mg i.v. t.i.d. One week later, the patient's Glasgow Coma Scale score dropped to 3 and the pupils became dilated. He was intubated and shifted to the Intensive Care Unit for mechanical ventilation support. Urgent CT of the brain was performed and revealed diffuse cerebral edema/diffuse global hypoxic ischemia. Brainstem reflexes and apnea test were conducted, and he was announced brain dead. The patient died 1 month later.

\section{Discussion}

In a multiethnic city like Dubai, NCC should be considered as one of the differential diagnoses for abnormal behavior. To the best of our knowledge, no other case report has been published regarding neuropsychiatric manifestations of NCC in this region.

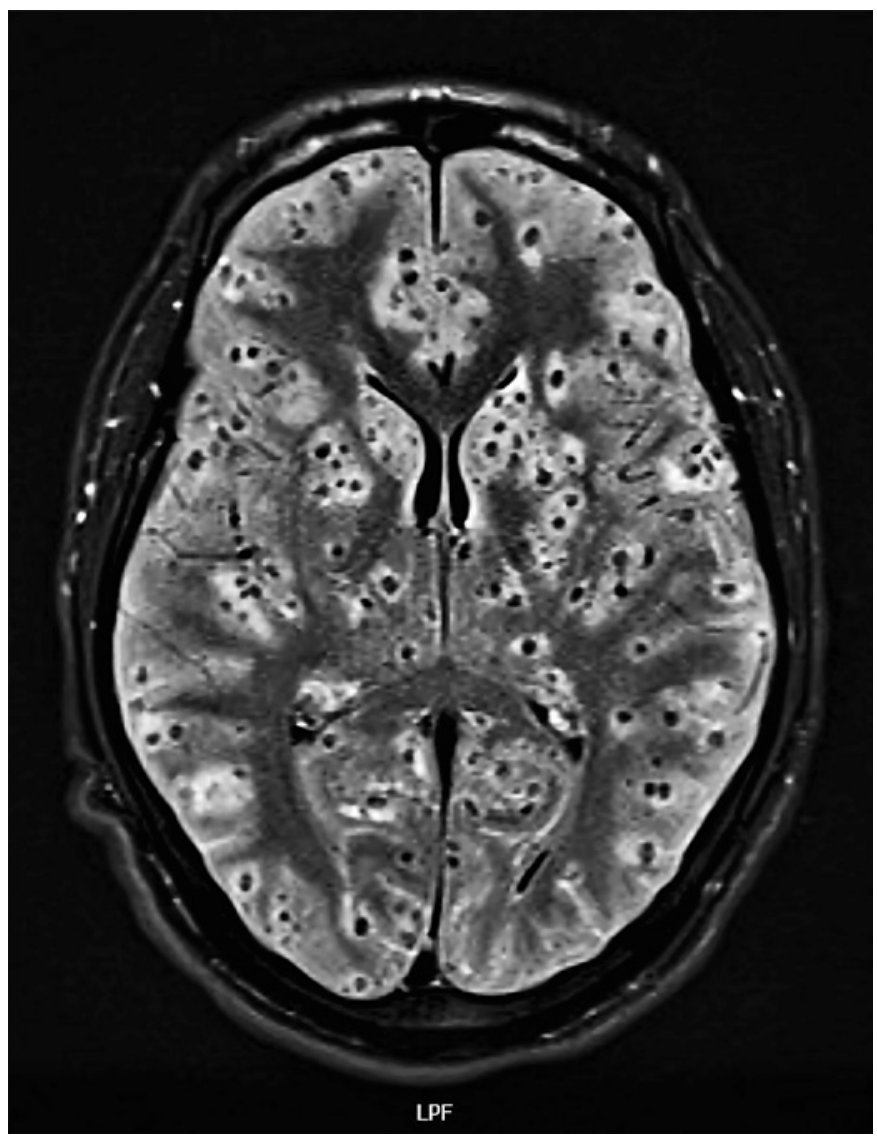

Fig. 1. MRI of the brain showing multiple ring-shaped lesions of NCC.

NCC is the most common neurological disease of parasitic origin. Neurological manifestations of NCC commonly present as seizures (80\%), headache (40\%), visual changes $(20 \%)$, and confusion (15\%). Certain immune mechanisms are initiated in the central nervous system, which cause reactive gliosis and arachnoiditis that ultimately lead to proliferative endarteritis. This causes occlusion of the arterial lumen and hydrocephalus, as a consequence of which there is intracranial hypertension and edema [8]. Such structural changes are heavily dependent on the quantity of parasitic lesions as well as their locations. In addition, ventricular and subarachnoid NCC are known to be associated with meningitis and intracranial hypertension, which can result in cognitive impairment, primarily attention deficit, decreased consciousness and delirium.

The diagnosis of NCC had frequently been delayed over the past decade due to an array of psychiatric manifestations predominating at the onset of the disease. For-
Mustafa/Al Ayyat/Awad/Elamin/ Bin Haider/Alkhoori 
lenza et al. [9] reviewed 38 cases of NCC which revealed psychiatric symptoms and cognitive decline in 65.8 and $87.5 \%$ of the cases, respectively. The most recurrent noncognitive psychiatric illness was identified as depression in $52.6 \%$ of the cases, whereas psychosis was identified in $14.2 \%$. Srivastava et al. [10] also revealed major depression and mixed anxiety depression as the two most common diagnoses, constituting 36 and $18 \%$ of the cases, respectively. Mahajan et al. [4] disclosed that their patient had had psychosis for 1 year before developing seizures and finally getting diagnosed as having NCC. In the present case report, the patient was oriented in the psychiatry ward but exhibited antisocial behavior, incoherent speech and suspicion. With substantial lack of history available regarding prior episodes of seizures or of epilepsy, it was unclear whether the patient was in a postictal state. Furthermore, no neurological signs or possible organic etiology indicated a suspicion of brief psychotic disorder.

Absolute criteria for the diagnosis of NCC constitute biopsy of the lesion to histologically demonstrate the parasite, visualization of the subretinal cysticercus and demonstration of the scolex within a cystic lesion on neuroimaging. Confirmative neuroimaging criteria also involve resolution of cystic lesions after cysticidal drug therapy [11]. The choice of treatment essentially depends upon the cyst viability, cysticercal load and location of cysts. For patients with 1-2 viable parenchymal cysticerci, albendazole $(15 \mathrm{mg} / \mathrm{kg} /$ day $)$ monotherapy for 10-14 days is recommended. More than 2 viable parenchymal cysticerci warrants albendazole with praziquantel $(50 \mathrm{mg} / \mathrm{kg} /$ day) for $10-14$ days [12]. Newer evidence has shown that commencement of antihelminthics can cause risk of exacerbated seizures and encephalopathy. This is primarily due to brain edema as a result of acute perilesional inflammation caused by cysts expelling toxic materials. This phenomenon usually occurs between the second to fifth day of therapy instigating larval death [13]. Thus, a course of steroids has actually improved patients' cognitive decline as it reduces inflammation.
Appropriate treatment also improves psychiatric symptoms. Psychiatric consultation should be offered for persistent psychiatric manifestations in patients and psychopharmacological therapy should be complimentary to neurological care.

\section{Conclusion}

It is highly recommended that NCC be considered as one of the possible differential diagnoses of abnormal behavior in multicultural cities, such as Dubai, where $71 \%$ of the total population constitutes expatriates from various countries.

\section{Statement of Ethics}

The patient in consideration was brought forth by the police with no identification documents in his possession. During his entire stay in the hospital, great attempts were made to locate the patient's family but no family or close relatives could be contacted. As the patient passed away, we were unable to obtain consent from him or any of his next of kin. The purpose of this case report is for academic benefit and to save lives, hence we deem it highly important that this case be published.

\section{Disclosure Statement}

The authors have no conflicts of interest to declare.

\section{Funding Sources}

The authors have no funding sources to declare.

\section{Author Contributions}

The corresponding authors analyzed medical records, laboratory and imaging reports to write the case report. PubMed was utilized to study papers on the topic at hand, with subsequent citations to strengthen the report.

The co-authors reviewed and adjusted the report with great consideration.

\section{References}

1 Del Brutto OH, Sotelo J. Neurocysticercosis: an update. Rev Infect Dis. 1988 Nov-Dec; 10(6):1075-87.

2 Román G, Sotelo J, Del Brutto O, Flisser A, Dumas M, Wadia N, et al. A proposal to declare neurocysticercosis an international reportable disease. Bull World Health Organ. 2000;78(3):399-406.
3 Pal DK, Carpio A, Sander JW. Neurocysticercosis and epilepsy in developing countries. J Neurol Neurosurg Psychiatry. 2000 Feb; 68(2):137-43

4 Mahajan SK, Machhan PC, Sood BR, Kumar S, Sharma DD, Mokta J, et al. Neurocysticercosis presenting with psychosis. J Assoc Physicians India. 2004 Aug;52:663-5.
5 Trivedi JK, Singh RK, Dalal PK. Psychiatric manifestations of cystecercosis : review of literature and case report. Indian J Psychiatry. 1983 Jan;25(1):74-7.

6 Aggarwal V, Kumar P, Chadda RK. Neurocysticercosis: uncommon presentation. Indian J Pediatr. 2001;68:34-5. 
7 Johnstone EC, Macmillan JF, Crow TJ. The occurrence of organic disease of possible or probable aetiological significance in a population of 268 cases of first episode schizophrenia. Psychol Med. 1987 May;17(2):371-9.

8 White AC Jr. Neurocysticercosis: updates on epidemiology, pathogenesis, diagnosis, and management. Annu Rev Med. 2000;51(1): 187-206.

9 Forlenza OV, Filho AH, Nobrega JP, dos Ramos Machado L, de Barros NG, de Camargo $\mathrm{CH}$, et al. Psychiatric manifestations of neu- rocysticercosis: a study of 38 patients from a neurology clinic in Brazil. J Neurol Neurosurg Psychiatry. 1997 Jun;62(6):612-6.

10 Srivastava S, Chadda RK, Bala K, Majumbar P. A study of neuropsychiatric manifestations in patients of neurocysticercosis. Indian J Psychiatry. 2013;55(3):264-67.

11 Del Brutto OH, Nash TE, White AC Jr, Rajshekhar V, Wilkins PP, Singh G, et al. Revised diagnostic criteria for neurocysticercosis. J Neurol Sci. 2017 Jan;372(15):20210.
12 White AC Jr, Coyle CM, Rajshekhar V, Singh G, Hauser WA, Mohanty A, et al. Diagnosis and Treatment of Neurocysticercosis: 2017 Clinical Practice Guidelines by the Infectious Diseases Society of America (IDSA) and the American Society of Tropical Medicine and Hygiene (ASTMH). Clin Infect Dis. 2018;66(8):e49-e75.

13 Das K, Mondal GP, Banerjee M, Mukherjee BB, Singh OP. Role of antiparasitic therapy for seizures and resolution of lesions in neurocysticercosis patients: an 8 year randomised study. J Clin Neurosci. 2007 Dec;14(12):1172-7. 